\title{
A continuum of weakly coupled oscillatory McKean neurons
}

\author{
M Denman-Johnson* and S Coombes ${ }^{\dagger}$ \\ Department of Mathematical Sciences, \\ Loughborough University, Leicestershire, LE11 3TU, UK.
}

(Dated: March 4, 2003)

\begin{abstract}
The McKean model of a neuron possesses a one dimensional fast voltage-like variable and a slow recovery variable. A recent geometric analysis of the singularly perturbed system has allowed an explicit construction of its phase response curve [S Coombes 2001 Phase-locking in networks of synaptically coupled McKean relaxation oscillators, Physica D, Vol 160, 173-188]. Here we use tools from coupled oscillator theory to study weakly coupled networks of McKean neurons. Using numerical techniques we show that the McKean system has traveling wave phase-locked solutions consistent with that of a network of more biophysically detailed Hodgkin-Huxley neurons.
\end{abstract}

*Electronic address: M.G.Denman-Johnson@lboro. ac .uk

${ }^{\dagger}$ Electronic address: S.Coombes@lboro.ac.uk 
Oscillatory behavior is observed in the normal functioning of various nerve cells. Guided by the celebrated work of Hodgkin and Huxley, mathematical descriptions based around systems of first order nonlinear differential equations are now commonly used to model such cells [1]. The investigation of these physiologically important models is typically hampered by both high dimensionality and nonlinearity. Apart from numerical analysis, progress in understanding has come about using geometric techniques [2-5] and tools from coupled oscillator theory [6-8]. By studying spiking neuron models, reduced by both geometric analysis and phase descriptions, one can begin to classify collective behavior in terms of physiologically significant parameters, such as those controlling the distributions of delays arising from synaptic processing. In this paper we pursue this approach for the McKean relaxation oscillator. Making extensive use of Fourier representations we show that a network of McKean neurons has dynamics consistent with that of the more biophysically detailed Hodgkin-Huxley model.

The equations for a single McKean oscillator take the form

$$
\begin{aligned}
\mu \dot{v} & =f(v)-w-w_{0}+I+\epsilon X(t) \\
\dot{w} & =v-\gamma w-v_{0},
\end{aligned}
$$

where the nonlinear function $f(v)$ is given by

$$
f(v)= \begin{cases}-v, & v<a / 2 \\ v-a, & a / 2<v<(1+a) / 2 \\ 1-v, & v>(1+a) / 2 .\end{cases}
$$

The variable $v$ corresponds to a membrane potential whilst $w$ is associated with the recovery property of a neuron. The parameters $a, \mu, w_{0}, v_{0}$ and $\gamma$ may be considered as combinations of membrane reversal potentials and conductance properties whilst $I$ is a constant input current. The term $\epsilon X(t)$ represents a time varying external input signal of strength $\epsilon$. In the absence of an time-varying input $(\epsilon=0)$ there is a range of $I$ values, determined by the condition that the (unstable) fixed point lie on the middle branch of $f(v)$, where the neuron can oscillate. In the singular limit $\mu \rightarrow 0$ the dynamics evolves on the invariant manifolds described by the left and right hand branches of the slow manifold $w=f(v)-w_{0}+I$. In this case, the period of oscillation is given by $T=T_{L}+T_{R}$ where $T_{L, R}$ are the times spent 
on the left (right) branches of the slow manifold and are easily calculated as

$$
\begin{aligned}
& T_{L}=\frac{1}{\beta} \ln \left[\frac{\left(-\beta w_{2}+A\right)}{\left(-\beta w_{1}+A\right)}\right], \\
& T_{R}=\frac{1}{\beta} \ln \left[\frac{\left(-\beta w_{1}+A+1\right)}{\left(-\beta w_{2}+A+1\right)}\right],
\end{aligned}
$$

where $\beta=1+\gamma, A=I-w_{0}-v_{0}, w_{1}=I-w_{0}-a / 2$ and $w_{2}=w_{1}+1 / 2$. Note that requiring the fixed point to be on the midle branch of $f$ is a sufficient condition for the existence of oscillations, but in the limit $\mu \rightarrow 0$ this condition becomes necessary. For weak coupling $(\epsilon \rightarrow 0)$ the dynamics of a single neuron can be reduced to a phase oscillator [6], which evolves according to

$$
\frac{\mathrm{d} \theta}{\mathrm{d} t}=\Omega+\epsilon R(\theta) X(t)
$$

Here $\Omega=1 / T$ and the phase response curve (PRC) $R(\theta)$ is given explicitly in [9] as

$$
R(\theta)-\widehat{R}(\theta)= \begin{cases}R_{1}(\theta)=\Omega \mathrm{e}^{\beta \theta T} /\left(A-\beta w_{2}\right), & \theta \in\left[0, \theta_{T}\right), \\ R_{2}(\theta)=B \mathrm{e}^{-\beta \theta_{T} T} R_{1}(\theta), & \theta \in\left(\theta_{T}, 1\right),\end{cases}
$$

where $\theta_{T}=T_{L} / T, B=\left(A-\beta w_{2}\right) /\left(A+1-\beta w_{1}\right)$, and

$$
\widehat{R}(\theta)=\kappa(0) \delta(\theta)+\kappa\left(\theta_{T}\right) \delta\left(\theta-\theta_{T}\right) .
$$

The constants $\kappa(0)$ and $\kappa\left(\theta_{T}\right)$ are chosen such that $\theta(t)$ evolves smoothly, ie $\kappa(0)=R_{1}(0)-$ $R_{2}(1)$ and $\kappa\left(\theta_{T}\right)=R_{2}\left(\theta_{T}\right)-R_{1}\left(\theta_{T}\right)$. The phase plane of the McKean model and a periodic relaxation orbit are depicted in Fig. 1 . Whenever the phase coordinate $\theta$ passes smoothly through $\theta_{T}$ an instantaneous jump of the system in the $(v, w)$ plane occurs and corresponds to the jump up between the slow manifolds. This signals the upswing of an action potential in the McKean model. Similarly, the downswing of an action potential occurs when $\theta$ increases through $\theta=1$ (with $\theta=1$ and $\theta=0$ identified) and there is a corresponding jump down between the slow manifolds.

To highlight the ability of the McKean model to caricature the behavior of the more biophysically complicated Hodgkin-Huxley model we consider recent work of Crook et al. [10-12] concerning the dynamics of a continuum model of weakly interacting phase oscillators. Interactions in their model are non-local and are represented as a spatial convolution involving some synaptic footprint kernel. Interestingly, they show that synchrony can be stable for localized excitatory coupling, but long range excitatory coupling leads to an unstable synchronous state. It is not our intention to explore any particular result of Crook et 


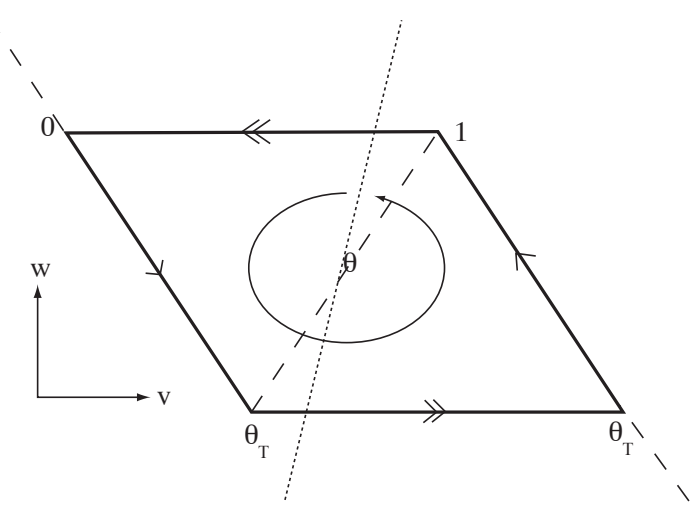

FIG. 1: Phase plane of the McKean model showing the type of periodic orbit (solid line) that occurs in the singular limit $\mu=0$. Note that the system jumps instantaneously from the left branch of the $\dot{v}=0$ nullcline (dashed line) to the right. The dotted line shows the linear nullcline defined by $\dot{w}=0$. In the phase coordinate the system evolves smoothly. The model is said to fire whenever $\theta$ passes through $\theta_{T}$.

al., but rather to illustrate that the McKean and Hodgkin-Huxley models have consistent network dynamics using the work of Crook et al. as a bench-mark. Their work focuses partly on sinusoidal phase interaction functions thought to provide a first approximation for more realistic Hodgkin-Huxley models and partly on the effects of synaptic processing (see [11] in particular). Here we pursue the analysis in [10-12] and pay close attention to the contributions from distributed synaptic delays along the lines described in [13]. We also present an explicit form for the phase interaction function that uses both the Fourier coefficients of the PRC and the Fourier coefficients of the synaptic response. This is different to the approach of Crook et al. who consider a Fourier expansion of the phase-interaction function from the outset and do not make direct use of the Fourier transform of the synaptic response function.

Following [11] we consider a continuous network of weakly coupled phase oscillators in one spatial dimension described by

$$
\frac{\partial \theta(x, t)}{\partial t}=\Omega+\int_{-\infty}^{\infty} W(y) H(\theta(x+y, t)-\theta(x, t)-|y| / c) \mathrm{d} y .
$$

The phase of the oscillator at position $x \in \mathbb{R}$ at time $t \in \mathbb{R}^{+}$is given by $\theta(x, t) \in[0,1)$. The term $|y| / c$ represents a space dependent delay arising from the finite propagation speed of signals (action potentials) between neurons. Note that $c$ represents the ratio of the signal 
velocity to the natural frequency of oscillation $\Omega$. In the weak coupling regime each oscillator approximately fires at its natural frequency $\Omega$. However, this relatively fast oscillation is slowly modulated by a drift in the phases induced by the non-local synaptic coupling to other neurons. The strength of interaction between oscillators a distance $y$ apart is specified by the synaptic footprint $W(y)$ which we shall take to be of exponential form $W(y)=\epsilon \exp (-|y|) / 2$. In obtaining the equation for the network phase dynamics it is assumed that $\epsilon$ is small and that the method of averaging applies (see for example [14]). Note that although the dynamics of the reduced phase model only shadows that of the true unaveraged system for times of $O\left(\epsilon^{-1}\right)$, hyperbolic orbits of the phase model do in fact correspond to hyperbolic orbits of the full system. We assume throughtout this paper that $\epsilon$ is fixed and small and from now on choose a synaptic strength scale where $\epsilon=1$. When describing a system of neural oscillators, the biological realism of the model typically resides in the phase interaction function $H(\theta)$. This can be expressed as the convolution of the PRC $R(\theta)$ with the synaptic input $P(t)$ :

$$
H(\theta)=\frac{1}{T} \int_{0}^{T} R(t / T) P(t+\theta T) \mathrm{d} t,
$$

where $T=\Omega^{-1}$. We refer the reader to [15] for further discussion about PRCs. The synaptic input is assumed to be $T$-periodic and of the form

$$
P(t)=\sum_{j} \eta(t+j T), \quad t \in[0, T)
$$

Here $\eta(t)$ describes the effects of synaptic processing and will be assumed to take the form of an alpha function $\eta(t)=\alpha^{2} t \exp (-\alpha t)$, for $t \geq 0$ and $\eta(t)=0$ for $t<0$. For ease of numerical calculation of phase interaction functions and PRCs we introduce a Fourier series representation for $P(t)$ :

$$
P(t)=\frac{1}{T} \sum_{k \in \mathbb{Z}} \widetilde{\eta}\left(\omega_{k}\right) \mathrm{e}^{\mathrm{i} \omega_{k} t}, \quad \omega_{k}=\frac{2 \pi k}{T} .
$$

Here $\widetilde{\eta}(\omega)$ is the (half) Fourier transform of $\eta(t)$ :

$$
\widetilde{\eta}(\omega)=\int_{0}^{\infty} \mathrm{e}^{-\mathrm{i} \omega t} \eta(t) \mathrm{d} t=\frac{\alpha^{2}}{(\alpha+\mathrm{i} \omega)^{2}} .
$$

Using the PRC of the McKean model we write the phase interaction function in the form

$$
H(\theta)-\widehat{H}(\theta)=B F\left(\theta ; 0,1-\theta_{T}\right)+\mathrm{e}^{\beta\left(\theta_{T}-1\right) T} F\left(\theta ; 1-\theta_{T}, 1\right)
$$


where

$$
F(\theta ; a, b)=\frac{1}{T} \int_{a}^{b} R_{1}(t) P(t+\theta T) \mathrm{d} t,
$$

(which is given in closed form in [9]) and

$$
\widehat{H}(\theta)=\Omega\left[\kappa(0) P\left(\left(\theta-\theta_{T}\right) T\right)+\kappa\left(\theta_{T}\right) P(\theta T)\right] .
$$

Introducing a Fourier series representation for $F(\theta ; a, b)=\sum_{k} F_{k}(a, b) \exp (2 \pi \mathrm{i} k \theta)$ :

$$
F_{k}(a, b)=\frac{\Omega^{3}}{A-\beta w_{2}} \frac{\widetilde{\eta}\left(\omega_{k}\right)}{\beta+\mathrm{i} \omega_{k}}\left[\mathrm{e}^{b\left(\beta+\mathrm{i} \omega_{k}\right)}-\mathrm{e}^{a\left(\beta+\mathrm{i} \omega_{k}\right)}\right]
$$

means that we may also write $H(\theta)=\sum_{k} H_{k} \exp (2 \pi \mathrm{i} k \theta)$ :

$$
\begin{aligned}
H_{k} & =B F_{k}\left(0,1-\theta_{T}\right)+\mathrm{e}^{\beta\left(\theta_{T}-1\right) T} F_{k}\left(1-\theta_{T}, 1\right) \\
& +\Omega^{2} \widetilde{\eta}\left(\omega_{k}\right)\left[\kappa(0) \mathrm{e}^{-2 \pi \mathrm{i} k \theta_{T}}+\kappa\left(\theta_{T}\right)\right] .
\end{aligned}
$$

When the PRC of an oscillator is not available in closed form it is always possible to obtain it numerically (see for example [7]). Moreover, the PRC of a real neuron can be found experimentally. In either case one may use a Fourier representation for the response function, $R(\theta)=\sum_{k} R_{k} \mathrm{e}^{2 \pi \mathrm{i} k \theta}$, where the coefficients $R_{k}$ are determined either from numerics or experiments. The phase interaction function then has Fourier coefficients given simply by

$$
H_{k}=\Omega \widetilde{\eta}\left(\omega_{k}\right) R_{-k}
$$

The usefulness of this approach arises from the adoption of a Fourier representation for both the PRC and the synaptic input current. In particular it allows one to deal with arbitrary synaptic kernels, for which the integral defining the phase interaction function cannot be done in closed form. Rather than resort to numerical quadrature the representation we use is an efficient alternative, especially if the Fourier coefficients of the synaptic response are known to decay fast (allowing early truncation of the sum over Fourier modes).

The phase oscillator network has a one parameter family of traveling wave solutions defined by $\theta(x, t)=\widetilde{\Omega} t+\beta x$, where

$$
\widetilde{\Omega}=\Omega+\int_{-\infty}^{\infty} W(y) H(\beta y-|y| / c) \mathrm{d} y
$$

A linear stability analysis shows that these solutions are stable if $\operatorname{Re} \lambda(p)<0$, where

$$
\lambda(p)=\int_{-\infty}^{\infty} W(y) H^{\prime}(\beta y-|y| / c)\left[\mathrm{e}^{\mathrm{i} p y}-1\right] \mathrm{d} y .
$$


Although the neutrally stable mode $\lambda(0)=0$ exists, it represents a perturbation by constant phase-shifts and so is excluded in the definition of linear stability. Using the property $W(y)=W(|y|)$ it is simple to show that

$$
\begin{aligned}
\lambda(p) & =\sum_{k} H_{k}^{\prime} \widehat{W}\left(p+2 \pi k \beta_{+}\right)-\widehat{W}\left(2 \pi k \beta_{+}\right) \\
& \left.+\widehat{W}\left(-p+2 \pi k \beta_{-}\right)-\widehat{W}\left(2 \pi k \beta_{-}\right)\right]
\end{aligned}
$$

where $\beta_{ \pm}= \pm \beta-1 / c, H_{k}^{\prime}=2 \pi \mathrm{i} k H_{k}$ and

$$
\widehat{W}(p)=\int_{0}^{\infty} W(y) \mathrm{e}^{\mathrm{i} p y} \mathrm{~d} y=\frac{1-\mathrm{i} p}{2} .
$$

The analysis of phase-locked and traveling wave states in networks of weakly coupled McKean relaxation oscillators can now be performed and compared with those in a corresponding Hodgkin-Huxley network. To ensure that the two models behave qualitatively the same we choose $v_{0}=w_{0}=0, I=0.5, \gamma=0.5$ and $a=0.32$ for the McKean model. This ensures that the duration of the action potential in each model is roughly the same as compared to their intrinsic period of oscillation. Note that it is the parameter $a$ which strongly influences the relative spike duration in this model and this is the one we have adjusted (compared to [9]) so as to bring the behavior of the McKean model more in line with that of the HodgkinHuxley model. Parameter values for the Hodgkin-Huxley model are the same as in [15] with an external drive of $I=20$. The PRC of the Hodgkin-Huxley model is obtained using XPP [16]. We fix an origin for the PRC of the Hodgkin-Huxley model by defining spike times to occur whenever the voltage variable increases through a threshold of $-57.4 \mathrm{mv}$. This is the point at which the PRC of the Hodgkin-Huxley model has its maximal value.

For fast rise times, in the sense that $\alpha / \Omega \rightarrow \infty$, we find that for both models the synchronous state $(\beta=0)$ is unstable for small $c$ and stable traveling waves appear. This is predicted using Fig. 2 where we present the stability regions of the traveling wave solutions in the $(\beta, c)$ parameter plane for the Hodgkin-Huxley and McKean models. The small discrepancy between the value of $c$ for which the synchronous solution changes stability can be traced back to the fact that the relative duration of action potentials between the two models is slightly different. Although the duration of the action potential in the McKean model can be adjusted further by varying $a$, this is not expected to lead to significant increased agreement. This is because, unlike the Hodgkin-Huxley model, the McKean model 


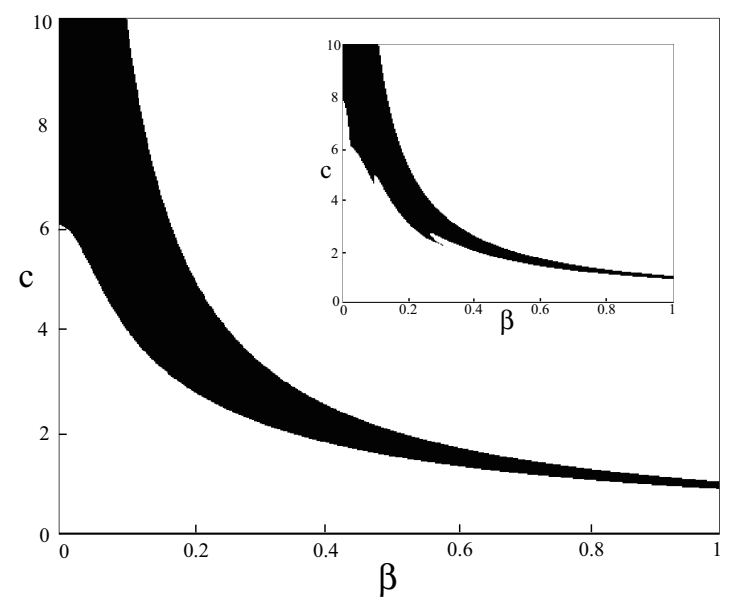

FIG. 2: Region of stability (black) for the traveling wave solution in the $(\beta, c)$ parameter plane for the continuum Hodgkin-Huxley model with an exponential synaptic footprint and an alpha function synaptic response with $\alpha=20 . c$ is the velocity of an action potential and $\beta$ is the phase gradient of a traveling wave: $\partial \theta(x, t) / \partial x=\beta$. The inset shows the same diagram for the McKean model. For low $c$ the synchronous solution $(\beta=0)$ is unstable and a stable traveling wave $(\beta \neq 0)$ can be found. A grid of $250 \times 250$ points is used.

is restricted to some singular limit $(\mu=0)$ where the rise and fall times of the action potential are instantaneous.

Crook et al. [11] have suggested that the change in stability of the synchronous solution as the propagation delay grows is consistent with the oscillatory behavior observed in both visual cortex, showing a tendency towards synchrony, and olfactory cortex, tending to produce traveling oscillatory waves; the latter has long-range connections and hence longer axonal delays. The stability diagrams for much slower synapses is produced in Fig. 3. Here, we see that both models once again give qualitatively similar predictions: namely that for slow synapses it is even easier to destabilize a synchronous solution in favor of a traveling wave. We note that the value of $c$ at which the synchronous solution changes from unstable to stable increases with decreasing $\alpha$. Also, the small amount of fine structure seen on the lower border of the McKean stability diagram (Fig. 2 inset) vanishes with increasing $\alpha$. This fine structure is related to the non-smooth behavior of the model seen in the singular limit $\mu=0$. 


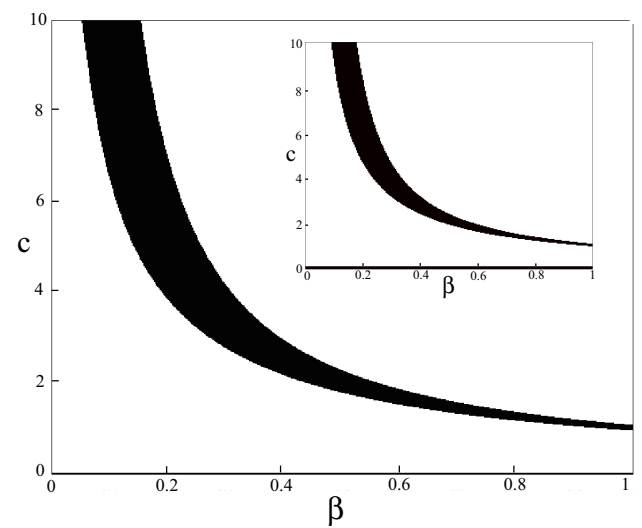

FIG. 3: Region of stability (black) for the traveling wave solution in the $(\beta, c)$ parameter plane for the continuum Hodgkin-Huxley model with an exponential synaptic footprint and an alpha function synaptic response with $\alpha=1$. The inset shows the same diagram for the McKean model. The value of $c$ at which the synchronous solution changes from unstable to stable increases with decreasing $\alpha$. A grid of $250 \times 250$ points is used.

In this paper we have shown that the dynamics of weakly interacting McKean relaxation oscillators captures the type of phase-locked behavior found in a corresponding HodgkinHuxley network. The assumption of weak coupling has allowed us to bring to bear the powerful machinery of coupled oscillator theory. For both models we have made use of Fourier techniques for the practical calculation of phase interaction functions. This has more readily allowed us to establish that phase-locked behavior of weakly coupled McKean and Hodgkin-Huxley networks is consistent. However, we should emphasize the two major assumptions of our analysis: strong relaxation and weak coupling. The PRC of the McKean model that we have used is valid only in the singular limit where the voltage variable operates on a much faster time scale than the recovery variable. However, by the Fenichel persistence theorem [17], results obtained in the singular limit $(\mu=0)$ are expected to extend to the non-singular limit ( $\mu$ small).

The authors would like to thank Steven Webb, Sarah Denman-Johnson and an anonymous referee for helpful comments made during the preparation of this manuscript. MDJ is supported by a research studentship from the Engineering and Physical Sciences Research 
Council (UK).

[1] A L Hodgkin and A F Huxley. A quantitative description of membrane current and its application to conduction and excitation in nerve tissue. Journal of Physiology (London), 116:449-472, 1952.

[2] D Somers and N Kopell. Rapid synchronization through fast threshold modulation. Biological Cybernetics, 68:393-407, 1993.

[3] D Terman and D Wang. Global competition and local cooperation in a network of neural oscillators. Physica D, 81:148-176, 1995.

[4] E M Izhikevich. Phase equations for relaxation oscillators. SIAM Journal on Applied Mathematics, 60:1789-1805, 2000.

[5] J Rubin and D Terman. Geometric analysis of population rhythms in synaptically coupled neuronal networks. Neural Computation, 12:597-645, 2000.

[6] G B Ermentrout and N Kopell. Frequency plateaus in a chain of weakly coupled oscillators. SIAM Journal on Applied Mathematics, 15:215-237, 1984.

[7] G B Ermentrout and N Kopell. Oscillator death in systems of coupled neural oscillators. SIAM Journal on Applied Mathematics, 50:125-146, 1990.

[8] G B Ermentrout and N Kopell. Multiple pulse interactions and averaging in systems of coupled neural oscillators. Journal of Mathematical Biology, 29:195-217, 1991.

[9] S Coombes. Phase locking in networks of synaptically coupled McKean relaxation oscillators. Physica D, 160:173-188, 2001.

[10] S M Crook. The role of delay in oscillatory models of olfactory cortex. PhD thesis, University of Maryland, 1996.

[11] S M Crook, G B Ermentrout, M C Vanier, and J M Bower. The role of axonal delay in the synchronization of networks of coupled cortical oscillators. Journal of Computational Neuroscience, 4:161-172, 1997.

[12] S M Crook, G B Ermentrout, and J M Bower. Dendritic and synaptic effects in systems of coupled cortical oscillators. Journal of Computational Neuroscience, 5:315-329, 1998.

[13] P C Bressloff and S Coombes. Physics of the extended neuron. International Journal of Modern Physics B, 11(20):2343-2392, 1997. 
[14] J Guckenheimer and P Holmes. Nonlinear Oscillations, Dynamical Systems, and Bifurcations of Vector Fields. Springer, 1983.

[15] B Ermentrout. Type I membranes, phase resetting curves, and synchrony. Neural Computation, 8:979-1001, 1996.

[16] B Ermentrout. Simulating, Analyzing, and Animating Dynamical Systems. SIAM, 2002. XPP software available from http://www.math.pitt.edu/ bard/xpp/xpp.html.

[17] N Fenichel. Persistence and smoothness of invariant manifolds for flows. Indiana University Mathematics Journal, 21:193-225, 1971. 